\title{
A rare case of Vieussens' arterial ring with multiple pulmonary fistulas and aneurysms
}

\author{
Duoyao Wang ${ }^{1,2}$, Chunhong $\mathrm{Hu}^{1}$ \\ ${ }^{1}$ Department of Radiology, The First Affiliated Hospital of Soochow University, Suzhou, China; ${ }^{2}$ Department of Radiology, Lianyungang Oriental \\ Hospital, Lianyungang, China
}

Correspondence to: Chunhong Hu. Department of Radiology, The First Affiliated Hospital of Soochow University Medical College, No. 899 PingHai Road, Suzhou 215006, China. Email: hch5030@163.com.

Submitted Oct 25, 2020. Accepted for publication Mar 07, 2021.

doi: 10.21037/qims-20-1197

View this article at: http://dx.doi.org/10.21037/qims-20-1197

\section{Introduction}

An abnormal connection between the conus branches of the right and proximal left anterior descending (LAD) coronary arteries is referred to as Vieussens' arterial ring (VAR). There are many pieces of VAR-related literature, including case reports and comments. However, the occurrence of VAR combined with pathological changes is extremely rare. Usually, VAR is not detected easily and patients do not present with any clinical symptoms. When a patient experiences symptoms such as chest tightness and chest pain, relevant imaging examinations may indicate this disease's existence. Here, we report a case of VAR with multiple pulmonary fistulas and aneurysms. After comparing various medical imaging examination methods, we believe that coronary computed tomography angiography (CCTA) is the most beneficial imaging method for displaying this complex disease.

\section{Case presentation}

A 55-year-old male patient was admitted to our hospital after experiencing symptoms of intermittent chest tightness with no apparent cause for 1 month. The patient had a history of hypertension and type 2 diabetes. His blood pressure and glycemia were well controlled. The electrocardiogram (ECG) showed ST-segment AVL depression and $\mathrm{rS}$ type in $\mathrm{V} 1-\mathrm{V} 3$. The patient's vital signs on admission showed a heart rate of 90 beats/minute, a body temperature of $36.4{ }^{\circ} \mathrm{C}$, a respiratory rate of 18 breaths/ minute, and blood pressure of 130/80 $\mathrm{mmHg}$. His B-type natriuretic peptide level and troponin level were $92.5 \mathrm{ng} / \mathrm{mL}$ (normal, 0-100 ng/mL) and $0.14 \mathrm{ng} / \mathrm{mL}$ (normal, $<0.01 \mathrm{ng} / \mathrm{mL}$ ), respectively. During admission, the patient reported sudden chest tightness, which was persistent and was not noticeably alleviated after rest. After the administration of nitroglycerin via an intravenous pump, the symptoms were relieved.

The patient subsequently underwent a series of medical imaging examinations. Coronary angiography revealed a coronary fistula from the LAD coronary artery's proximal portion to the main pulmonary artery. The proximal LAD coronary artery branched tortuously and displayed an aneurysm formation (Figure $1 A, B, C, D$ ). Thus, more anatomical information was needed to obtain further data to inform the next treatment. The patient underwent examination with CCTA. The three-dimensional volumerendered reconstruction revealed that the conus branches of the right coronary artery and the proximal portion of the LAD coronary artery formed a VAR. The LAD coronary artery's proximal branches were tortuous, and closely adhered to and extended into the pulmonary artery to include a pulmonary fistula; they were also locally complicated with an aneurysm formation. The coronary artery fistula (CAF), VAR, and aneurysm are displayed in the reconstructed CCTA images in Figure 2.

\section{Discussion}

VAR situation were first proposed and described by Raymond de Vieussens in 1706 (1), who later unified them 

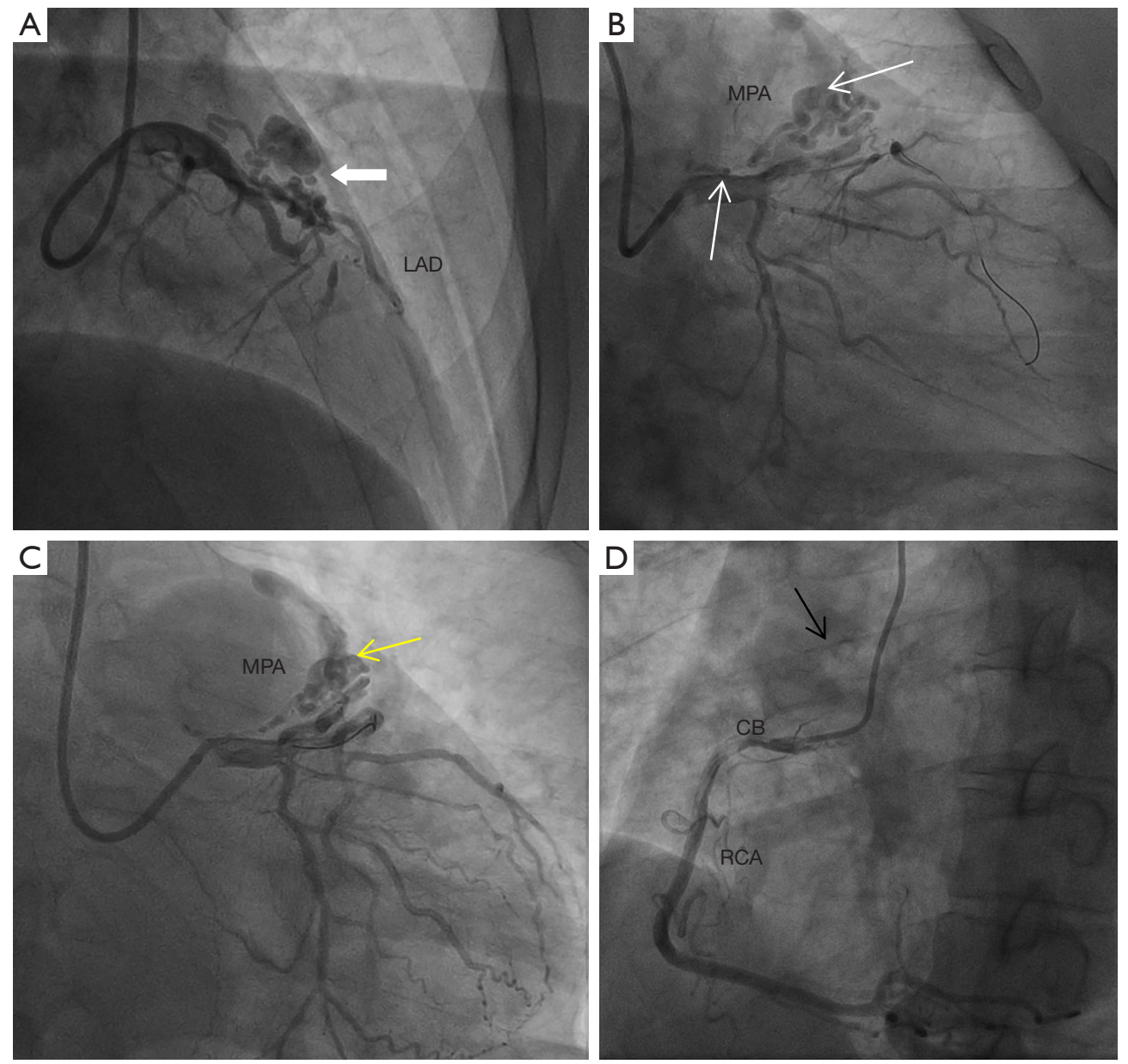

Figure 1 Coronary angiography images showing the fistula from the coronary artery to the main pulmonary artery using different views. An aneurysm (white arrow) is shown in the tortuous branch of the proximal LAD coronary artery (A). The CAF (thin white arrow) draining into the MPA (B), and worm-like tortuous and tubular dilated fistula vessels (thin yellow arrow) attached over the MPA (C). The distal vessel of the CB (thin black arrow) running in the LAD coronary artery direction, suggesting the possibility of VAR (D). MPA, main pulmonary artery; LAD, left anterior descending; RCA, right coronary artery; $\mathrm{CB}$, conus branches of the right coronary artery; CAF, coronary artery fistula; VAR, Vieussens' arterial ring.

as the VAR. When 1 of the left or right coronary arteries has a disease, such as stenosis, the structure of the VAR will expand its flow to the diseased side (the side with the lower pressure). This structure can ensure the blood supply to a certain extent. Doğan et al. (2) found that the detection rate of VAR to be only $3.19 \%$ o through imaging studies. Moreover, in that study, the authors divided VAR into 4 types according to the different definitions of the VAR variants (Table 1). When the VAR has an accompanying aneurysm, fistula, or other vascular pathological condition, it is considered to be type 1B. Although Doğan et al. defined this type, no type $1 \mathrm{~B}$ cases were found in their research.
The case reported in the present paper fits this rare type 1B VAR. The proportion of coronary artery fistulas that occur in patients with congenital heart disease is $0.2 \%$ to $0.4 \%$. Compared with genetic and acquired factors, congenital dysplasia is relatively common (3). Sakakibara et al. (4) classified coronary fistulas into 5 types depending on the fistula's location (Table 2). Drainage of the fistula into the pulmonary artery, which was described in the present case, belongs to type III. Lee et al. found that multisource fistulas caused the mixing of multiple blood flow sources to produce tremendous pressure, which increased the risk of combined aneurysm (5). 

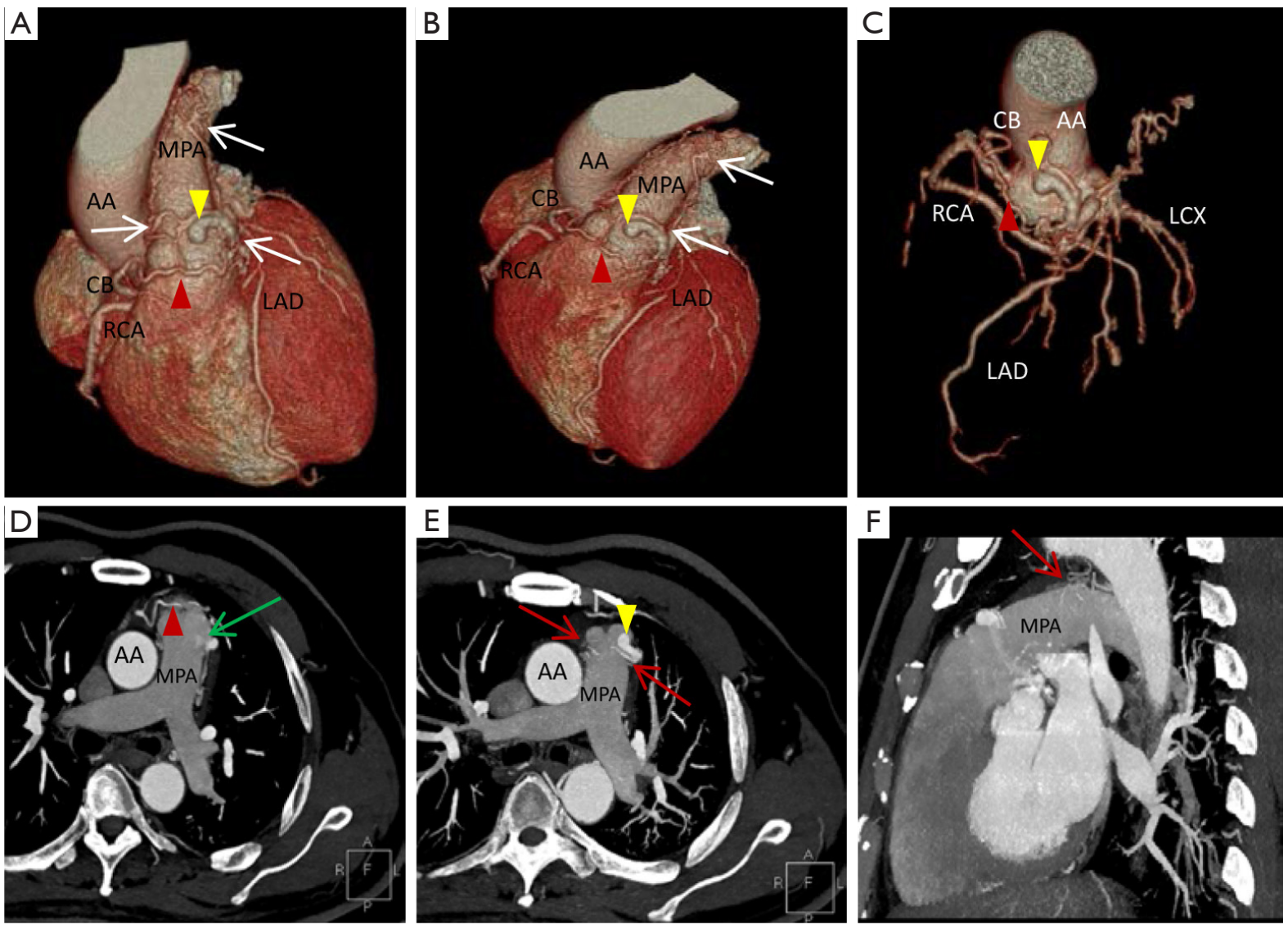

Figure 2 Three-dimensional volume-rendered CCTA reconstructions (A-C). The connection between the CB and the proximal LAD coronary artery forming a VAR (red arrowhead). Worm-like tortuous and tubular fistula vessels attached over the MPA; the thin white arrows show interconnections between these vessels. Dilated fistula vessels accompanied by aneurysm formation (yellow arrowhead). Axial and sagittal MIP images showing multiple CAFs (D-F). A large CAF drainage site with contrast media into the left lateral wall of the MPA shown on axial MIP images (thin green arrow). Multiple small CAF drainage sites are visible in the different lateral walls of the MPA (thin red arrow). AA, ascending aorta; MPA, main pulmonary artery; LAD, left anterior descending artery; RCA, right coronary artery; CAF, coronary artery fistula; VAR, Vieussens' arterial ring; CCTA, coronary computed tomography angiography; MIP, maximum intensity projection.

Table 1 The classification of VAR variants (2)

\begin{tabular}{ll}
\hline Type & VAR variants \\
\hline 1A & Without accompanying vascular pathology \\
$1 B$ & With accompanying vascular pathology (aneurysm/fistula) \\
2 & VAR-like dual LAD duplication \\
3 & With single coronary artery anomaly \\
\hline
\end{tabular}

VAR, Vieussens' arterial ring; LAD, left anterior descending artery.
Table 2 The classification of coronary artery fistulas (4)

\begin{tabular}{ll}
\hline Type & Explanation \\
\hline I & Drainage into the right atrium \\
II & Drainage into the right ventricle \\
III & Drainage into the pulmonary \\
IV & Drainage into the left atrium \\
V & Drainage into the left ventricle \\
\hline
\end{tabular}


The treatment of asymptomatic CAFs in adults with no significant hemodynamic changes is controversial (6). The patient described above had palpitations, dyspnea, and other symptoms that required further treatment. When considering therapeutic interventions for CAF, surgical and interventional procedures should be considered. Indications for interventional therapy to close the fistula include light fistula distortion, a single fistula, and no other cardiovascular malformations. Surgery is suitable for patients with large shunts, multiple fistulas, tortuous expansion of the fistula, and aneurysms, as well as for those who cannot undergo interventional therapy (7). The main surgical techniques include surgical ligation alone, coronary aneurysm resection, and surgical ligation with coronary artery bypass grafting. In the present case, the patient was suitable for surgical treatment. However, the patient's symptoms were relieved, and conservative treatment was temporarily used for follow-up observation. The patient now uses antiplatelet drugs, calcium channel blockers, and nitrates daily to prevent thrombosis and relieve his symptoms. In the next stage, the patient will require further surgery. Because the conus branches of the right coronary artery and multiple blood vessels in the LAD coronary artery proximal part were twisted into clusters, this patient's VAR presentation was not clear on coronary angiography (Figure 1D). CCTA can accurately display the origin of the $\mathrm{CAF}$, drainage location, fistula situation, and the presence or absence of associated aneurysms, to guide the selection of appropriate treatment options. It can also aid in the accurate evaluation of coronary arteries in severely ill infants (8). CCTA should be used as the preferred imaging method for the clinical diagnosis and follow-up of CAFs.

\section{Acknowledgments}

The authors thank Dr. Yubao Wang, Dr. Xishan Yi, and Dr. Suyan Zhang for their valuable suggestions.

Funding: None.

\section{Footnote}

Conflicts of Interest: Both authors have completed the ICMJE uniform disclosure form (available at http://dx.doi. org/10.21037/qims-20-1197). The authors have no conflicts of interest to declare.

Ethical Statement: We have obtained written informed consent from this patient to publish the relevant medical images and research report.

Open Access Statement: This is an Open Access article distributed in accordance with the Creative Commons Attribution-NonCommercial-NoDerivs 4.0 International License (CC BY-NC-ND 4.0), which permits the noncommercial replication and distribution of the article with the strict proviso that no changes or edits are made and the original work is properly cited (including links to both the formal publication through the relevant DOI and the license). See: https://creativecommons.org/licenses/by-nc-nd/4.0/.

\section{References}

1. Loukas M, Clarke P, Tubbs RS, Kapos T. Raymond de Vieussens. Anat Sci Int 2007;82:233-6.

2. Doğan N, Dursun A, Özkan H. Vieussens' arterial ring: A rare coronary variant anatomy. Diagn Interv Radiol 2019;25:109-13.

3. Said SA. Congenital solitary coronary artery fistulas characterized by their drainage sites. World J Cardiol 2010;2:6-12.

4. Sakakibara S, Yokoyama M, Takao A, Nogi M, Gomi H. Coronary arteriovenous fistula. Am Heart J 1966;72:307-14.

5. Lee CM, Song SY, Jeon SC, Park CK, Choi YW, Lee Y. Characteristics of Coronary Artery to Pulmonary Artery Fistula on Coronary Computed Tomography Angiography. J Comput Assist Tomogr 2016;40:398-401.

6. Loukas M, Germain AS, Gabriel A, John A, Tubbs RS, Spicer D. Coronary artery fistula: a review. Cardiovasc Pathol 2015;24:141-8.

7. Saboo SS, Juan YH, Khandelwal A, George E, Steigner ML, Landzberg M, Rybicki FJ. MDCT of congenital coronary artery fistulas. AJR Am J Roentgenol 2014;203:W244-52.

8. Roik D, Kucińska B, Roik M, Werner B. Ring of Vieussens: A Collateral Coronary Pathway on ElectrocardiographyGated 320-row CT in a 10-week-old Boy With the Anomalous Left Coronary Artery From the Pulmonary Artery. Kardiol Pol 2020;78:603-4.

Cite this article as: Wang D, Hu C. A rare case of Vieussens' arterial ring with multiple pulmonary fistulas and aneurysms. Quant Imaging Med Surg 2021;11(10):4504-4507. doi: 10.21037/qims-20-1197 